\title{
Investigating the Effects of Hydrophobicity and Charge on the Therapeutic Ability of the Antimicrobial Histatin 8 Peptide for Potential Use in Oral Applications
}

\author{
Scott M. G. Matheson ${ }^{1}$, Naowarat Cheeptham ${ }^{2}$ \& Heidi E. K. Huttunen-Hennelly ${ }^{1}$ \\ ${ }^{1}$ Department of Physical Sciences (Chemistry), Thompson Rivers University, BC, Canada \\ ${ }^{2}$ Department of Biological Sciences, Thompson Rivers University, BC, Canada \\ Correspondence: Heidi E. K. Huttunen-Hennelly, Department of Physical Sciences (Chemistry), Thompson \\ Rivers University, 900 McGill Road, Kamloops, BC V2C 0C8, Canada. Tel: 1-250-377-6063. E-mail: \\ hhuttunen@tru.ca
}

Received: January 23, 2013 Accepted: February 27, 2013 Online Published: March 13, 2013

doi:10.5539/ijb.v5n2p85 URL: http://dx.doi.org/10.5539/ijb.v5n2p85

\begin{abstract}
Advances in technology allow for the construction of synthetic antibiotics, which includes the development of de novo antimicrobial peptides (AMPs). The contents of AMPs including amino acids, chain length, hydrophobicity, ring structure/rigidity, terminus and charge when modified can alter the antimicrobial properties. A special family of peptides called histatins is naturally excreted by oral glands as an immune response, and previous research shows their potential for treating thrush. Histatin 8 is known to have antimicrobial activity against yeast strains and the goal of this study was to synthesize histatin 8 and two novel derivatives ( $\Delta 1$ and $\Delta 4)$ that fall at extremes of each other with regard to charge and hydrophobicity in order to investigate the properties that could optimize antimicrobial properties. The derivatives were characterized using various chemical and biological assays to investigate the effects of charge and hydrophobicity on bioactivity. Compared to histatin $8, \Delta 4$ 's minimum inhibitory concentration (MIC) was decreased more than tenfold against Candida tropicalis indicating increased antimicrobial activity. Re-inoculation confirmed fungicidal properties.
\end{abstract}

Keywords: antimicrobial peptides, histatin 8, peptide bioactivity

\section{Introduction}

The discovery of antibiotics has changed the way medicine is practiced, and has been the answer to many bacterial and fungal infections over the past several generations. Antibiotics are one of the most frequently prescribed medications in modern time, and despite careful precautions, have readily been over-prescribed leading to multiple antibiotic resistant microorganisms (pathogens) (Bonomo \& Szabo, 2006). The present demand of antibiotics has consequently made the search of natural antibiotics impractical, and with no major new discoveries since 1970 until 2000 (Clatworthy, Pierson, \& Hung, 2007), and an ever increasing resistance to conventional antibiotics, innovated approaches for developing antibiotics is called for (Hancock, 1997; Wilcox, 2004).

Advances in technology have allowed the construction of a relatively novel class of drugs called antimicrobial peptides (AMPs), which includes the development of de novo AMPs. Underlying elements of native AMPs such as chain length (Deslouches et al., 2005), hydrophobicity (Avrahami, Oren, \& Shai, 2001), ring structure/rigidity (Fernandez-Lopez et al., 2001), amino acids (D/L) (Deslouches et al., 2005), terminus (Raguse, Porter, Weisblum, \& Gellman, 2002), and charge (Hancock, 1997) can be modified to alter antimicrobial activity, thus therapeutic properties. Synthetic AMPs, in order to be useful, need to be designed such that they maximize antimicrobial activity without causing toxicity to humans. An advantage of AMPs is that they are far less likely to acquire resistance, as they deploy rapid modes of action, making them excellent candidates as novel therapeutic agents (Yeaman \& Yount, 2003). AMPs have shown activity towards Gram positive and negative bacteria, fungi, viruses, and even cancer cells (Hale \& Hancock, 2007). Once an AMP-host membrane interaction has occurred, modes of action can include transmembrane pore formation and gradient dissolution and/or intracellular binding to molecules such as DNA and RNA (Nicolas, 2009). 
As a growing body of literature on AMPs continues to accumulate, many fundamental principles of synthetic design are becoming well established to improve the therapeutic potential of peptides, which can potentially meet all the requirements of an effective antibiotic. The process of modifying a naturally occurring peptide into derivatives is one approach for developing new AMPs with predicted selectivity. Histatin 8 (KFHEKHHSHRGY), found in human saliva, possesses activity against fungi (Table 1) (Wang, 2003). This peptide is of interest as there is limited research on novel histatin derivatives. Histatin 8 has a significant role in immune function as it naturally prevents oral thrush, which is active when natural mouth flora like Candida albicans, Candida glabrata, and Candida tropicalis grow in excess. Thrush creates white sores in the mouth and allows entry of bacteria into the blood stream and vital organs; having damaging effects (Takakura et al., 2003). Patients with diabetes, cancer, HIV or other chronic diseases that result in a weakened immune system are at the highest risk of thrush because natural levels of histatin are diminished. Current treatment is antifungal medication, but in many cases it is incompatible with other medications ( $\mathrm{Su}, \mathrm{Gaskie,} \mathrm{\&} \mathrm{Jamieson,} \mathrm{2008).}$ Therefore, due to its natural occurrence in the oral cavity histatin 8 and synthetic derivatives present a low chance of toxicity. Creating histatin 8 derivatives with increased antimicrobial activity could have useful applications in conventional mouthwash or oral hygiene products.

Table 1. Histatin 8 characteristics and improvements

\begin{tabular}{lll}
\hline & Reference Peptide & Ideal Improvement \\
\hline Name & Human Histatin 8 & N/A \\
Source & Homo sapiens & N/A \\
Sequence & KFHEKHHSHRGY & *Substitute Histidine with Alanine \\
Length & 12 & 12 \\
Structure & Rich in Histidine & Rich in Alanine \\
Activity & Fungi & Candida causing oral candidiasis \\
Net charge & 6 & Decreasing \\
Hydrophobic residues & 8 & Increasing \\
\hline
\end{tabular}

\section{Methods}

\subsection{General}

To confirm peptide identity, samples were prepared with the addition of $0.1 \%$ formic acid and dissolved in a 1:1 ratio of methanol and water and analyzed at the University of Alberta, Canada by electrospray Ionization mass spectrometry using a Finnigan LCQ Advantage Mass Spectrometer at $5 \mu \mathrm{L} / \mathrm{min}$ flow rate with selective-ion storage. Peptide concentrations after vacufuging were determined using a Quant-iT Assay kit. The kit included concentrated assay reagents, dilution buffers, and pre-diluted BSA standards. Absorbance was recorded on the Quant-iT Fluorometer, and peptide concentration was determined from the linear calibration curve and peptide absorbance.

\subsection{Peptide Synthesis and Purification}

An Applied Biosystems 431A automated peptide synthesizer was used to synthesize the peptides using standard Fmoc chemistry, and all reagents were reagent grade. The peptides were synthesized on a $0.8 \mathrm{mmol}$ scale using FastMoc ${ }^{\mathrm{TM}}$ protocols and Fmoc protected amino acids, solvents and coupling reagents were purchased from Advanced Chemtech (Louisville, KY, USA). Each amino acid coupling cycle was approximately 60 minutes and includes Fmoc deprotection using piperidine, washing using N-methylpyrrolidone (NMP), coupling step to 1.0 mmol of the next Fmoc amino acid using 2-(1H-Benzotriazole)1,1,3,3-tetramethyluronium hexafluorophosphate (HBTU) and 1-hydroxybenzotriazole (HOBt) as coupling reagents, and lastly wash with NMP. The peptides were cleaved from the solid phase using a cleavage mixture $(9.0 \mathrm{~mL}$ TFA, $0.5 \mathrm{~mL}$ thioanisole, $0.3 \mathrm{~mL}$ EDT, and $0.2 \mathrm{~mL}$ anisole) at room temperature for 4 hours (the reaction was initially cooled in an ice bath for 5 minutes). The mixture was quantitatively transferred using the remaining cleavage solution onto a medium pore glass-fritted filter and washed with dichloromethane (DCM) under vacuum. The mixture was transferred to a separatory funnel and extracted using three aliquots of deionized water and one back extraction using ether to help remove impurities from the water layer. Using a high-pressure rotary evaporation apparatus and a water 
bath at $40^{\circ} \mathrm{C}$, the organic solvents were removed and the water layer was reduced. Final purification of the peptides was performed using a Waters PrepLC 400 system $(250 \mathrm{~mm}$ x $21.20 \mathrm{~mm}$ x $10 \mu \mathrm{m}$ particle size, 300 Åpore size) C18 reversed-phase column with $3 \mathrm{~mL}$ samples injections with detection at $220 \mathrm{~nm}$ and manual collection. Prior to injection the peptides were filtered through a $0.45 \mu \mathrm{M}$ Nylon ${ }^{\mathrm{TM}}$ syringe filter, and then run at a flow rate of $10 \mathrm{~mL} / \mathrm{min}$ using helium sparged filtered water $(0.1 \%$ TFA $) / H P L C$-grade acetonitrile $(0.05 \%$ TFA) gradient. Acetonitrile was removed using high-pressure rotary evaporation with a $40{ }^{\circ} \mathrm{C}$ water bath. The $\%$ yield and characterization by Mass Spectrometry (MS) is seen in Table 2.

Table 2. Yields (\%) of purified peptides and MS characterization of histatin 8 and alanine-based derivatives

\begin{tabular}{lll}
\hline Peptide & \% Yield & Mass (Da.) \\
\hline Histatin 8 & 81 & 1562 \\
$\Delta 1$ & 73 & 1496 \\
$\Delta 4$ & 74 & 1298 \\
\hline
\end{tabular}

*Calculated masses are within 1 Da.

\subsection{Antimicrobial Activity}

Fungal cultures were grown by inoculating Yeast Mold (YM) broth medium with one isolated colony from either Candida albicans, Candida tropicalis, or Candida glabrata. These three specific fungi were used as they are known to cause oral thrush and be susceptible to histatin 8 . To verify mid log phase, growth time courses were determined by periodically sampling the culture inoculated at $37^{\circ} \mathrm{C}$ every approximately 30 minutes using a spectrophotometer at $600 \mathrm{~nm}$. Furthermore, cultures were grown to the same turbidity with a 0.5 McFarland standard (O.D. $\left.{ }_{600}=0.319\right)$ on a shaking incubator for 4 hours at $37^{\circ} \mathrm{C}$ at $175 \mathrm{rpm}$.

To perform a Kirby Bauer assay, the fungal broths were then diluted to appropriate turbidity to ensure consistent colony forming units $\left(\mathrm{CFU}=1.5 \times 10^{5} \mathrm{CFU} / \mathrm{mL}\right)$ for all Kirby Bauer plates. A total of $200 \mu \mathrm{L}$ of the appropriate fungal grown to mid log phase was spread by hockey stick method on YM plates and left to dry in a flow hood for 10 minutes. To prepare an impregnated disk with the peptide, paper discs $(6 \mathrm{~mm}$ in diameter, Toyo Adventec, Japan) were placed on a glass plate, impregnated with $20 \mu \mathrm{L}$ of a specific concentration of peptide and let to air dry. The impregnated disks then were placed on the prepared YM plates. The plates were incubated at $37^{\circ} \mathrm{C}$ for 24 hours and observed, according to the standard protocol. Zones of inhibition were recorded as positive (zones of inhibition) or negative (no zones of inhibition) to preliminary determine antimicrobial activity. Gram positive Methicillin-Resistant Staphylococcus aureus and Gram negative Escherichia coli were subjected to a Kirby Bauer assay using the above described protocols except that the YM broth was replaced with Mueller Hinton (MH) broth.

Minimum Inhibitory Concentration (MIC) assays were performed on peptides that showed positive Kirby Bauer results. The peptides were made up to known concentrations using serial dilutions including $5 \times 10^{-3} \mathrm{~g} / \mathrm{mL}, 1 \times 10^{-3}$ $\mathrm{g} / \mathrm{mL}, 1 \times 10^{-4} \mathrm{~g} / \mathrm{mL}$, and $1 \times 10^{-5} \mathrm{~g} / \mathrm{mL}$. The same fungal species (Candida albicans, Candida tropicalis, and Candida glabrata) were inoculated into YM nutrient broth containing the known peptides dissolved in aqueous media. The prepared solutions were then incubated at $37^{\circ} \mathrm{C}$ with mixing at $175 \mathrm{rpm}$ overnight. Optical density at $600 \mathrm{~nm}$ was recorded to determine inhibitory effects, where a 90\% decrease in OD compared to the peptide-free controls was recorded as the MIC. The spectrophotometer was blanked using the YM media broth, negative control consisted of the broth, microbe, and no peptide, while the positive control consisted of broth, microbe, and a known antimicrobial agent (Nystatin). The negative control demonstrates optimal bacterial growth, and the positive control demonstrated aseptic technique as no growth should be seen. All samples were prepared in triplicate. The inhibitory concentration was expressed as a percent of growth inhibited (Brown, 1988), and expressed as:

$$
\text { MIC } / \% \text { Active Concentration }=100-(\text { sample/positive control }) \times 100
$$

\section{Results}

\subsection{Peptide Design}

An improved AMP is determined predominantly on enhanced antimicrobial activity, thus the goal of this project was to increase the derivative activity compared to histatin 8 in order to develop potential therapeutic agents. When developing derivatives, parameters including net charge, helicity, hydrophobicity per amino acid $(\mathrm{H})$, 
hydrophobic moment of the total peptide $(\mu)$, and the angle subtended by the positively charged polar helix face (ø) could be manipulated (Matsuzaki, 2009). Two parameters (charge and hydrophobicity) were modified in the parent peptide histatin 8 . By substituting alanine residues in exchange for positively charged histidine residues, we were interested in studying the change (if any) in antimicrobial activity thus providing evidence that hydrophobicity and charge influence this property. Histatin 8 is a great candidate for this study because it is a small peptide that can be easily synthesized and it has a significant proportion of histidine residues that can be replaced. Histatin peptides are also naturally found in the human oral cavity and derivatives theoretically have a low chance of producing any harmful hemolytic effects. While there have been previous studies concerning histatin effects on yeast, studies investigating the change in charge and hydrophobicity have yet to be examined. Furthermore, an online secondary structure predictor (NetSurfP ver. 1.1) determined all peptides were random coil, and derivatives were developed to reduce significant change in structure. The histatin 8 derivatives (Table 3) were synthesized and examined holistically.

Table 3. Peptide library with histatin 8 sequence and peptide derivatives with abbreviations and estimated literature values

\begin{tabular}{llll}
\hline Sequence & Abbreviations & Total hydrophobic ratio (\%) & Total net charge \\
\hline KFHEKHHSHRGY & Histatin 8 & 8 & +6 \\
KFHEKHHSARGY & $\Delta 1$ & 16 & +5 \\
KFAEKAASARGY & $\Delta 4$ & 41 & +2
\end{tabular}

Note: Single letter amino acid abbreviations: Lysine (K), Phenylalanine (F), Histidine (H), Glutamine acid (E), Serine (S), Arginine (R), Glycine (G), Tyrosine (Y), Alanine (A). Derivative $\Delta 1$ and $\Delta 4$ corresponds to substitution of one and four histidines for alanines, respectively.

Since histatin 8 contains four histidines amino acids out of its 12 amino acid sequence, alanine was substituted once $(\Delta 1)$ and then four times $(\Delta 4)$ in place of the cationic histidine residues to make two novel derivatives that fall at extremes of each other with regard to charge and hydrophobicity. The $\Delta 1$ derivative was altered specifically at the last histidine position in order to retain known molecular interactions between specific amino acids. The parent histatin 8 peptide, and many other peptides, form specific structures such as cationic amphipathic helices that are responsible for their therapeutic properties. Changing the peptide sequence may jeopardize the integrity of the native secondary structure and potentially eliminate activity. For instance, it's been previously noted that the helicity of histatin peptides are stabilized by the motif lysine-arginine-lysine-phenylalanine-histidine-glutamic acid-lysine-histidine (K-R-K-F-H-E-K-H) (Ramalingam, Gururaja, Ramasubbu, \& Levine, 1996). Consequently, $\Delta 1$ was designed to keep that motif intact such that the new derivative, $\Delta 1$, should remain bioactive.

Successful synthesis of histatin 8 and the two derivatives (the least hydrophobic derivative, $\Delta 1$, with a total hydrophobic ratio percentage (THR) of 16 , and most hydrophobic derivative, $\Delta 4$, with a THR 41) allowed for the investigation of hydrophobicity/charge effects on bioactivity and hemolysis. The bioactivity of the three peptides against selected fungi and bacteria was investigated.

\subsection{Antimicrobial Activity}

After subjecting the peptides to Kirby Bauer assays to preliminarily determine the presence of antimicrobial activity, the MICs listed in Table 4 were determined. The native peptide and one derivative $(\Delta 4)$ were active in destroying and inhibiting fungal growth, which is imperative for comparison and discussion regarding the effects of hydrophobicity and charge on peptide design. 
Table 4. MICs of peptides against fungal pathogens. MICs or Active concentrations $(\mathrm{g} / \mathrm{mL})$ are determined by a reduced optical density of at least $90 \%$ compared to control culture without peptide, and a (-) indicated no activity at the concentrations tested

\begin{tabular}{llll}
\hline & \multicolumn{3}{c}{ Minimal Inhibitory Concentrations $(\mathrm{g} / \mathrm{mL})$} \\
\hline Peptide & Candida albicans & Candida tropicalis & Candida glabrata \\
Histatin 8 & $(1.0 \pm 0.1) \times 10^{-3}$ & $(5.0 \pm 0.2) \times 10^{-3}$ & - \\
$\Delta 1$ & - & - & - \\
$\Delta 4$ & $(7.5 \pm 0.2) \times 10^{-3}$ & $(1.0 \pm 0.2) \times 10^{-4}$ & - \\
\hline
\end{tabular}

The $\mathrm{MIC}_{90}$ was used to demonstrated antimicrobial activity instead of total lysis, as it is common for high concentrations of peptide to cause total depletion in $\mathrm{OD}_{600}$ measured for growth compared to the negative control; and lower concentrations close to the MIC yielding absorbance's near the initial inoculum $\mathrm{OD}_{600}$ that plateaus for a length in time. Furthermore, the viable inoculum at $\mathrm{MIC}_{90}$ was re-inoculated in growth media containing no peptide, confirming the presence of fungicidal as opposed to simply fungistatic properties. Results show a narrow spectrum of activity toward Candida (in particular C. albicans and C. tropicalis), as there was no activity against additional tests on gram positive Methicillin-Resistant Staphylococcus aureus and gram negative Escherichia coli bacteria.

\section{Discussion}

A substance that destroys a microbe by membrane disruption and membrane penetration is indicative of both trans-membrane and intracellular mechanisms, which may be related to the hydrophobicity of the peptide derivative. Histatin 8 is a known anti-fungal peptide, yet its mode of action remains to be a controversial topic. With a net positive charge and low hydrophobicity it has previously been shown to have membranolytic activity by causing depolarization of membranes (Johnson, Yeh, \& Dodds, 2000). To start selective facilitation of microbe membrane disruption, it is said that a strong electrostatic interaction between polar phospholipid head groups of a cell membrane and the cationic face of an AMP must transpire. ${ }^{19}$ Future studies may determine whether cell death is then facilitated by the binding of the peptide to a permeable surface and using a combination of its hydrophobic and hydrophilic regions that aggregate themselves into the membrane where they may act extracellularly by forming pores or by displacing the lipid bilayer by means of the barrel stave, carpet, or toroidal pore mechanism or by acting intracellularly by binding to molecules inside the cell such as DNA and RNA (Hancock \& Sahl, 2006).

Given the low hydrophobicity and small size of histatin 8 , it seems unlikely to penetrate the polymeric fibrils of fungal cell walls and be permeable to the amorphous matrix components. Moreover, its weak amphiphilic properties also suggest that direct insertion is the favorable mode of action rather than ion channel formation. Tauro and Coutinho further investigated possible mechanisms and demonstrated that the native confirmation of histatin 8 is most commonly $\beta$-pleated sheet but adopts an $\alpha$-helix structure when placed in different solvents that closely mimic cellular environment's (Taruo \& Coutinho, 2002). Thus, to facilitate improved antimicrobial activity it was hypothesized that increasing the hydrophobicity of histatin 8 derivatives would allow for improved membrane interaction with hydrophobic phospholipids for entry to the membrane interior for initial ion channel formation, as well as dissociation from the membrane into the cytosol to act intracellularly.

There may have been many reasons as to why derivative $\Delta 4$ had improved activity over the reference peptide histatin 8 including the following. Movement through the phospholipid bilayer was facilitated by the addition of four hydrophobic alanine residues which increased the THR by 33\%. Removing the larger basic polar histidines for the smaller neutral nonpolar alanine residues may have also increased molecular plasticity needed for $\Delta 4$ 's mechanism of action. Furthermore, the consequential decrease in net positive charge did not terminate activity, therefore supporting the importance of hydrophobicity for bioactivity. Conversely, the lack of activity for derivative $\Delta 1$ may have resulted from a variety of reasons. For example, due to limited studies regarding important structural motifs, a disruption of the amino acid sequence may have affected intramolecular and intermolecular interactions. $\Delta 1$ only had a THR of $16 \%$, and coupled with an altered sequence, may not have been hydrophobic enough to compensate for the small loss in positive charge.

\section{Conclusion}

Histatin 8 and two novel derivatives were successfully designed, synthesized and characterized to show the significance of hydrophobicity/charge on antimicrobial activity and therefore therapeutic potential. The rich-alanine derivative, $\Delta 4$, had an increase in the THR of $33 \%$ compared to the native histatin 8 , while 
experimental results showed a decrease in MIC. The decrease in MIC (increase in antimicrobial activity) may be due to improved membrane interaction with hydrophobic phospholipids for entry into the membrane interior and also dissociation from the membrane into the cytosol. Furthermore, molecular plasticity of $\Delta 4$ may have enabled access to different conformations that were necessary for its mechanism of action. Furthermore, an active concentration of $1 \times 10^{-4} \mathrm{~g} / \mathrm{mL}$ makes $\Delta 4$ a realistic candidate as a therapeutic agent, yet further tests need to be conducted to determine in vivo potential that investigate hemolytic activity using such experiments as cell culture assays. As a result, the experimental results support the notion that substituting in hydrophobic amino acids into derivatives can increase their therapeutic antimicrobial activity despite reducing its cationic charge.

Future work may include using other methods to manipulate the peptide by introducing D-amino acids or by cyclization of the linear peptide. Incorporating amino acids that are not readily recognized by bacteria may help with resistance, while cyclization would remove the peptide termini which should make proteolytic degradation by enzymes more difficult. In some cases cyclization can increase the therapeutic properties by increasing antimicrobial activity and decreasing hemolytic activity (Manivannana \& Sukumar, 2007). Experimental design of such a proposed study may be reproduced using methods described by Wessolowski and colleagues, as they investigated the effects of cyclization and introducing D-amino acids (Wessolowski, Bienert, \& Dathe, 2004). It was reported that select linear peptides displayed moderate and no activity towards Gram positive and negative bacteria, while cyclization provoked high antimicrobial activity. The incorporation of D-amino acids had minor influence on the antimicrobial activity and resulted in a low hemolytic activity. To explain the possible mechanism along with the membrane stability and ability to induce membrane depolarization a calcein leakage assay for the calcein-entrapped vesicles by the derivatives may be done.

\section{Acknowledgements}

We would like to express our gratitude to the Comprehensive University Enhancement Fund at Thompson Rivers University (TRU) for funding the project. In addition, acknowledgments are given to the Departments of Physical and Biological Sciences at TRU, D. Nelson and R. Smith for their guidance, and C. Fardy for her microbiology laboratory expertise and training, ethics approval, and blood donations.

\section{References}

Avrahami, D., Oren, Z., \& Shai, Y. (2001). Effect of multiple aliphatic amino acids substitutions on the structure, function, and mode of action of diastereomeric membrane active peptides. Biochemistry, 40, 12591-12603. http://dx.doi.org/10.1021/bi0105330

Bonomo, R., \& Szabo, B. (2006). Mechanisms of Multidrug Resistance in Acinetobacter Species and Pseudomonas aeruginosa. Clinical Infectious Diseases, 43, 49-56. http://dx.doi.org/10.1086/504477

Brown B. A. (1988). Hematology: Principles and Procedures (5th ed.). Philadelphia, PA: Lea \& Febiger, pp. $139-142$.

Clatworthy, A., Pierson, E., \& Hung, D. (2007). Targeting virulence: a new paradigm for antimicrobial therapy. Nature Chemical Biology, 3, 541-548. http://dx.doi.org/10.1038/nchembio.2007.24

Deslouches, B., Phadke, S. M., Lazarevic, V., Cascio, M., Islam, K., Montelaro, R. C., \& Mietzner, T. A. (2005). De novo generation of cationic antimicrobial peptides: Influence of length and tryptophan substitution on antimicrobial activity. Antimicrobial Agents and Chemotherapy, 45, 316-322. http://dx.doi.org/10.1128/AAC.49.1.316-322.2005

Fernandez-Lopez, S., Kim, H. S., Chol, E. C., Delgado, M., Granja, J. R., Khasanov, A., ... Ghadirl, M. R. (2001). Antibacterial agents based on the cyclic D,L-alpha-peptide architecture. Nature, 412, 452-456. http://dx.doi.org/10.1038/35086601

Hale, J., \& Hancock, R. E. W. (2007). Alternative mechanisms of action of cationic antimicrobial peptides on bacteria. Expert Review of Anti-Infective Therapy, 5, 951-959. http://dx.doi.org/10.1586/14787210.5.6.951

Hancock, R. E. W. (1997). Peptide antibiotics. Lancet, 349, 418-423. http://dx.doi.org/10.1016/S0140-6736(97)80051-7

Hancock, R. E. W., \& Sahl, H. G. (2006). Antimicrobial and host-defense peptides as new anti-infective therapeutic strategies. Nature Biotechnology, 24, 1551-1557. http://dx.doi.org/10.1038/nbt1267

Johnson, D. A., Yeh, C. K., \& Dodds, M. W. J. (2000). Effect of donor age on the concentrations of histatins in human parotid and submandibular/sublingual saliva. Archives in Oral Biology, 45, 731-740. http://dx.doi.org/10.1016/S0003-9969(00)00047-9 
Manivannana, R., \& Sukumar D. (2007). The RBC membrane stabilization in an in vitro method by the drug isolated from Leucas aspera. International Journal of Applied Science and Engineering, 133, 133-138.

Matsuzaki, K. (2009). Control of cell selectivity of antimicrobial peptides. Biochimica Et Biophysica Acta-Biomembranes, 1788, 1687-1692. http://dx.doi.org/10.1016/j.bbamem.2008.09.013

Nicolas, P. (2009). Multifunctional host defense peptides: intracellular-targeting antimicrobial peptides. FEBS Journal, 276, 6483-6496. http://dx.doi.org/10.1111/j.1742-4658.2009.07359.x

Raguse, T., Porter, E., Weisblum, B., \& Gellman, S. (2002). Structure-activity studies of 14-helical antimicrobial beta-peptides: Probing the relationship between conformational stability and antimicrobial potency. Journal of the American Chemical Society, 124, 12774-12785. http://dx.doi.org/10.1021/ja0270423

Ramalingam, K., Gururaja, T. L., Ramasubbu, N., \& Levine, M. J. (1996). Stabilization of helix by side-chain interactions in histatin-derived peptides: Role in candidacidal activity. Biochemical Biophysical Research Communications, 225, 47-53. http://dx.doi.org/10.1006/bbrc.1996.1129

$\mathrm{Su}, \mathrm{C}$. , Gaskie, S., \& Jamieson, B. (2008). What is the best treatment for oral thrush in healthly infants? Journal of Family Practice, 57, 484-485.

Takakura, N., Sato, Y., Ishibashi, H., Oshima, H., Uchida, K., \& Yarnaguchi, H. (2003). A novel murine model of oral candidiasis with local symptoms characteristic of oral thrush. Microbiology and Immunology, 47, 321-326.

Tauro, S., \& Coutinho, E. (2002). Conformation of the peptide antibiotic - histatin 8 in aqueous and non-aqueous media. Letters in Peptide Science, 8, 295-307. http://dx.doi.org/10.1007/BF02538356

Wang, S. (2003). The Antimicrobial Peptide Data Base. Eppley Institute. Retrieve January 21, 2013, from http://aps.unmc.edu/AP/database/query_output.php?ID=00523

Wessolowski, A., Bienert, M., \& Dathe, M. (2004). Antimicrobial activity of arginine- and tryptophan-rich hexapeptides: the effects of aromatic clusters, d-amino acid substitution and cyclization. Journal of Peptide Research, 64, 159-169. http://dx.doi.org/10.1111/j.1399-3011.2004.00182.x

Wilcox, S. (2004). The new antimicrobial cationic peptides. BioTeach Journal, 2, 88-91.

Yeaman, M., \& Yount, N. (2003). Mechanisms of Antimicrobial Peptide Action and Resistance. Pharmacological Reviews, 55, 27-55. http://dx.doi.org/10.1124/pr.55.1.2 